\title{
Documents pour servir à la connaissance de la faune de Mammifères des grottes du Banat (Roumanie)
}

\author{
Par Alexandrina Negrea, Lazare Botoşăneanu, Ştefan Negrea ${ }^{1}$ )
}

Au cours des années 1960-1965 les auteurs ont effectué des recherches de caractère complexe dans les grottes du Banat. Ils ont rassemblé à cette occasion de riches matériaux de Mammifères actuels et fossiles dans plus de 70 grottes. Dans les pages qui suivront nous allons faire mention exclusivement des matériaux recueillis par nous mêmes, ainsi que de nos propres observations; tout ceci pourra profitablement compléter ce que d'autres auteurs avaient antérieurement réalisé dans cette région karstique.

Nous pensons, en disant ceci, à: Balogh, Bokor, Calinescu, Dumitrescu-Tanasachi et Orghidan, Halaváts, Hamar, Hoernes, Kadič, Maxim, Méhely, Nicolaescu-Plopşor et Mateescu, Orthmayr, Petényi, Paszlawski, Primics, Sencu, Teglas, Terzea (voir la bibliographie) ainsi qu'à d'autres auteurs.

Mentionnons que les pièces ostéologiques ont été recueillies soit en surface des dépôts de remplissage, soit en pratiquant des fouilles très superficielles, en remuant le matériel d'anciennes fouilles ou bien en examinant les restes de repas de carnassiers ou les ingluvies des Oiseaux. Une véritable fouille n'a jamais été pratiquée. De plus, on a toujours fait des captures de Chiroptères (les ectoparasites trouvés sur ceux-ci seront mentionnés) et on a pris note de toutes les observations possibles sur les autres Vertébrés rencontrés.

Toutes les grottes figurant dans la «Liste des grottes dans lesquelles des Mammifères ont été collectés ou observés», portent un numéro d'ordre qui sera utilisé dans la «Liste des espèces identifiées». D'autre part, presque toutes les grottes de notre liste portent, entre parenthèses, un numéro correspondant à celui du Répertoire des grottes de Roumanie figurant sur la carte des régions carpatiques de Roumanie (Orghidan et collab., 1965). Les grottes, peu nombreuses, ne figurant

1) Institutul de Speologie «Emil Rakovitza»», Str. Dr. Capşa nr. 8, Bucuresti 35 (Roumanie). 
pas dans ce Répertoire, pourront facilement être mises en place sur la carte, grâce à leur position dans notre Liste; on pourra trouver une présentation détaillée de presque toutes les grottes dans Botoşaneanu, Negrea et Negrea (1966) et dans Negrea, Negrea, Sencu et Botoşaneanu (1965).

Le matériel est gardé dans les collections de l'Institut de Spéologie «Emile Racovitza» de Bucarest.

Il nous est fort agréable d'adresser nos remerciements pour des déterminations et pour de précieux renseignements, aux personnes suivantes: Prof. Dr. Margareta Dumitrescu (Rodentia p.p., Insectivora, Chiroptera), Dr. Elena Terzea (Carnivora p.p., Lagomorpha, Rodentia p.p.), Dr. C. Radulescu et P. Samson (Carnivora p.p., Artiodactyla), Anca Burghele (Nycteribiidae) et Maria Georgescu (Ixodidae).

\section{Liste des grottes dans lesquelles des Mammifères ont été collectés ou observés}

Grottes situées dans le bassin supérieur de la Bega

1. P. $\left.{ }^{2}\right)$ de la Româneşti (590).Vulpes oulpes; Ursus arctos; Ursus spelaeus; Capra? Ovis ?; Apodemus sylsaticus; Myotis myotis; Miniopterus schreibersi.

2. P. Gaura din Cioaca Birtului (598). Glis glis; Myotis mystacinus.

3. Galeria de explorare din Valea cu Baia. Rhinolophus ferrum-equinum; Miniopterus schreibersi. Autres Vertébrés: larves de Salamandra maculosa.

\section{Grottes situées dans le bassin du Pogăniş}

4. P. Casa Lotrilor (506). Rhinolophus hipposideros.

Grottes situées dans le bassin de la Bîrzava au S et au SE de Reşița

5. P. Stîrnic (508). Ursus spelaeus; Cerous elaphus; Rupicapra rupicapra; Glig glis; Clethrionomys glareolus; Aroicola terrestris; Sorex minutus ?; Talpa europaea; Rhinolophus blasii; Rhinolophus ferrum-equinum; Rhinolophus hipposideros.

6. P. cu oase de la Stîrnic (509). Vulpes vulpes; Ursus spelaeus; Muscardinus avellanarius.

7. P. Gaura de la Capu Baciului (510). Vulpes sulpes; Martes foina; Sus scrofa (domesticus?); Rupicapra rupicapra; Microtus arealis; Apodemus sylsaticus; Crocidura leucodon; Rhinolophus hipposideros.

8. P. Gaura Pîrşului de la Capu Baciului (511). Canis familiaris; Ursus spelaeus; Sus scrofa (domesticus ?); Glis glis; Rhinolophus ferrum-equinum; Rhinolophus hipposideros.

9. P. Gaura Turcului (512). Rhinolophus ferrum-equinum; Miniopterus schreibersi.

10. P. Gaura Pîrşului (513). Sus scrofa (domesticus?); Rhinolophus mehelyi.

2) P. = abbréviation de »Peştera» (Grotte). 
Grottes situées dans le bassin supérieur du Caraş

11. P. Cîrnealǎ (535). Vulpes sulpes; Capreolus capreolus; Apodemus sylsaticus; Chiroptera (obs., indet.).

12. P. Buhui (536). Ursus spelaeus; Rhinolophus ferrum-equinum; Myotis myotis; Myotis oxygnathus.

13. P. Cuptoare (537). Glis glis; Myotis oxygnathus; Rhinolophus ferrumequinum; Rhinolophus hipposideros; Myotis myotis.

14. P. Salamandrelor. Aucun Mammifère. Autres Vertébrés: Salamandra maculosa.

15. P. nr. 2 de la Haldinǎ (540). Rhinolophus hipposideros.

16. P. Cerbului (516). Ursus spelaeus; Clethrionomys glareolus; Microtus aroalis; Apodemus sylvaticus; Myotis myotis; Myotis mystacinus. Tous les restes squelettiques de Rongeurs ont été trouvés dans l'ingluvie d'un Oiseau.

17. P. Popovăț (517). Ursus spelaeus; Felis sylvestris; Capreolus capreolus; Glis glis; Cricetulus migratorius; Apodemus sylvaticus; Clethrionomys glareolus; Rhinolophus ferrum-equinum; Rhinolophus hipposideros; Myotis myotis; Myotis becksteini; Myotis emarginatus; Myotis mystacinus; Barbastella barbastellus; Eptesicus nilssoni. Fort nombreux restes squelettiques de Chiroptères et de Rongeurs, surtout dans les zones stalagmitées et sèches. De nombreuses pièces sont même recouvertes d'une croûte ou inclues dans la croûte de calcite.

18. P. Comarnic (520). Rhinolophus ferrum-equinum; Rhinolophus hipposideros; Myotis myotis; Myotis oxygnathus.

19. P. din Valea Topliței. Lepus europaeus.

20. P. Racoviță. Vormela peregusna; Martes foina; Felis syloystris; Rupicapra rupicapra; Capra? Ovis ?; Glis glis; Muscardinus avellanarius; Clethrionomys glareolus; Microtus arealis; Microtus sp.; Apodemus sylsaticus; Myotis capaccini; Rhinolophus ferrum-equinum. Dans les niches latérales sèches et même dans la galerie principale, plusieurs "cimetières", agglomérations de restes squelettiques de Chiroptères et de Rongeurs.

21. P. de la Socolovǎț (518). Des os longs d'Artiodactyles (indet.) et des restes squelettiques de Rana sp. et d'Oiseaux ont été observés.

22. P. nr. 1 de sub Crno Pole. Capra? Ovis ? Chiroptera (obs., indet.).

23. P. nr. 2 de sub Crno Pole. Ursus arctos; Myotis capaccinii; Rhinolophus hipposideros.

24. P. Tolosu (522). Rhinolophus ferrum-equinum; Myotis myotis; Myotis oxygnathus; Myotis capaccinii; Myotis emarginatus; Pipistrellus pipistrellus.

25. P. din Drumul Prolazului (523). Rhinolophus hipposideros.

26. P. cu Fereastră (524). Glis glis; Chiroptera (obs., indet.).

27. P. de sub Cetate I (525). Cricetulus migratorius; Microtus?

28. P. de sub Cetate II (526). Aucun Mammifère. Autres Vertébrés: Cinclus cinclus (nidifiant dans la grotte).

29. P. Liliecilor (527). Felis cf. catus; Glis glis; Myotis myotis; Myotis capaccinii; Miniopterus schreibersi; Plecotus austriacus?

30. P. de după Cîrşa (530). Vulpes vulpes; Sus scrofa (domesticus ?); Glis glis; Myotis myotis; Pipistrellus pipistrellus; Rhinolophus ferrum-equinum.

31. P. Spinului (531). Rhinolophus ferrum-equinum.

32. P. Lizlonea. Aucun Mammifère. Autres Vertébrés: Salamandra maculosa.

33. P. de sub peştera Vraşka. Vulpes oulpes; Crocuta spelaea. 
34. P. nr. 2 din Cureacița. Crocuta spelaea; Felis catus; Apodemus sylvaticus.

35. P. nr. 3 din Cureacița. Sciurus vulgaris.

36. P. nr. 5 din Cureacița. Rhinolophus ferrum-equinum.

37. P. nr. 6 din Cureacița. Aucun Mammifère. Autres Vertébrés: Vipera berus berus (vivante).

38. P. Gaura lui Schimpfin (541). Ursus spelaeus; Clethrionomys glareolus; Sorex araneus; Rhinolophus blasii; Rhinolophus mehelyi. Autres Vertébrés: Natrix sp.

39. P. Galațiului (533). Meles meles.

40. P. cu apă din Cheile Gîrliştei (534). Microtus arvalis; Apodemus sylvaticus; Rhinolophus ferrum-equinum; Rhinolophus euryale; Rhinolophus blasii; Myotis myotis; Miniopterus schreibersi.

\section{Grottes creusées dans la montagne Rolul Nou (Giclova Montană)}

41. P. de sub Padina Popii (542). Capra? Ovis ?; Apodemus sylsaticus; Glis glis; Microtus?; Talpa europaea.

42. Avenul de la Fîntîna lui Ilie. Meles meles.

\section{Grottes situées dans le bassin du Miniş entre ses sources et Poneasca}

43. P. Ponor-Plopa (543). Rhinolophus ferrum-equinum. Autres Vertébrés: Salmo trutta $m$. fario, dans le ruisseau souterrain.

44. P. Ponor-Uscatǎ (544). Myotis oxygnathus.

45. P. Găurile lui Miloi II (550). Miniopterus schreibersi.

\section{Grottes situées dans le bassin du Lăpuşnic}

46. P. de la Vălee (553). Ursus spelaeus; Glis glis; Clethrionomys glareolus; Apodemus syloaticus; Spallax microphthalmus; Myotis myotis; Myotis oxygnathus; Rhinolophus ferrum-equinum; Rhinolophus hipposideros; Rhinolophus mehelyi. De nombreux restes squelettiques sont inclus dans le plancher stalagmitique ou bien recouverts d'une croûte de calcite.

\section{Grottes situées dans les gorges de la Nera}

47. P. Voinii (559). Rhinolophus ferrum-equinum.

48. P. lui Vît (560). Vulpes vulpes; Felis (Lynx) lynx; Ursus spelaeus; Capra ibex; Apodemus sylvaticus ?; Rhinolophus ferrum-equinum; Rhinolophus hipposideros.

49. P. Boilor (562). Rhinolophus ferrum-equinum; Rhinolophus hipposideros.

50. P. Mare de la Găuri (564). Ursus spelaeus; Sus scrofa (probablement sauvage); Sciurus vulgaris; Glis glis; Rhinolophus hipposideros.

51. P. Gaura Porcariului (565). Ursus spelaeus; Capreolus capreolus; Capra hircus; Glis glis; Rhinolophus ferrum-equinum; Myotis oxygnathus.

52. P. Dubova (566). Ursus spelaeus; Canis familiaris; Meles meles; Capreolus capreolus; Apodemus sylvaticus; Glis glis; Muscardinus avellanarius; Clethrionomys glareolus; Microtus arvalis; Pithymys subterraneus; Sciurus 
oulgaris; Crocidura leucodon; Talpa europaea; Myotis oxygnathus; Myotis myotis; Rhinolophus hipposideros; Rhinolophus ferrum-equinum; Rhinolophus blasii; Plecotus auritus.

53. P. Rolului (567). Rhinolophus blasii.

Grottes situées dans le bassin du Danube, à l'E. et au SE de Moldova Nouă

54. P. Ieskinia (568). Rhinolophus hipposideros.

55. P. Filipovo Dira (569). Vulpes vulpes; Chiroptera (obs., indet.)

56. P. de la Padina Matei (570). Mustela putorius; Felis sylvestris; Glis glis; Rhinolophus ferrum-equinum; Rhinolophus blasii; Miniopterus schreibersi.

57. P. Gaura Haiduceascǎ (571). Glis glis; Muscardinus avellanarius; Clethrionomys glareolus; Microtus arvalis; Apodemus sylsaticus; Sorex? Crocidura leucodon ?; Talpa europaea; Rhinolophus ferrum-equinum; Rhinolophus hipposideros. Sur la "terrasse» supérieure de la grande salle, importante agglomération de restes squelettiques de Chiroptères, Rongeurs et Insectivores, œuvre probablement d'un Mammifère de proie.

58. P. din Valea Čeuca (573). Felis sylvestris; Microtus arvalis; Rhinolophus ferrum-equinum; Rhinolophus blasii.

59. P. de la Izvoru Mînzului (576). Rhinolophus hipposideros.

60. P. U Lomu (578). Microtus arsalis; Apodemus sylvaticus.

61. P. Potoc (579). Vulpes oulpes.

62. P. Gaura cu Muscă (580). Myotis myotis; Myotis emarginatus; Miniopterus schreibersi; Rhinolophus ferrum-equinum; Rhinolophus blasii.

\section{Grottes creusées dans la Vallée de Berzasca}

63. P. de la Voinicovăț (496). Ovis ? Capra ?; Myotis emarginatus; Miniopterus schreibersi; Eptesicus serotinus.

64. P. din Cleanțu Pînzei (498). Rhinolophus ferrum-equinum.

65. P. din Cleanțu Zbegului (499). Vulpes oulpes; Glis glis; Cricetulus migratorius; Microtus arvalis.

66. P. Zamonița (500). Lutra lutra; Crocidura leucodon.

\section{Grottes creusées dans le massif Sucaru Mare}

(Défilé du Danube de Cazane)

67. P. nr. 2 de la Gura Ponicovei (493). Capra? Ovis ?; Rhinolophus hipposideros.

68. P. Gura Ponicovei (494). Vulpes oulpes; Crocuta spelaea; Mustela putorius; Martes martes; Martes foina; Ursus spelaeus; Capreolus capreolus; Ovis ? Capra ?; Glis glis; Clethrionomys glareolus; Apodemus sylvaticus; Myotis myotis; Nyctalus noctula; Plecotus auritus; Barbastella barbastellus; Pipistrellus pipistrellus; Eptesicus serotinus; Rhinolophus blasii. Autres Vertébrés: Tichodroma muraria, dans l'intérieur de la grotte, à quelques dizaines de mètres de l'entrée débouchant sur le Danube.

69. P. din Pînza Curii (Veterani) (495). Pipistrellus pipistrellus. Nous avons noté aussi des restes squelettiques de: Ursus sp., Mustellidae, Ovis, mais nous n'avons pas la certitude de ces déterminations. 
Grottes situées dans le bassin inférieur de la Cerna

70. P. Hoților (485). Rhinolophus blasii; Myotis myotis.

71. P. Mare de la Soronişte (488). Ursus spelaeus; Myotis oxygnathus.

72. P. Gaura Ungurului (491). Ursus spelaeus; Clethrionomys glareolus; Apodemus sylvaticus; Rhinolophus ferrum-equinum; Rhinolophus blasii; Myotis myotis.

\title{
Liste des espèces déterminées, avec observations $\left.{ }^{3}\right)$
}

\author{
Supraord. CARNIVORA (ord. FISSIPEDES)
}

\section{Fam. CANIDAE}

Canis familiaris L. 8 (max. sin. fragm.) 52 ( $\mathrm{M}^{2} \sin ., \mathrm{D}^{2-4} \sin$. , Cd $\sup$. $\sin$.).

Vulpes sulpes (L.). $\mathbf{1}$ (mdb.). 6 (crâne, cubitus, tibia). $\mathbf{7}$ (crâne juvénile). 11 (I, arc mdb.). 30 (max. sup. dextr. fragm.). 33 (fragm. mdb. sin.). 48 ( $\mathrm{P}^{2-}$ $\mathrm{M}^{2}$ dextr., $\mathrm{M}^{2}$ sin.). 55 ( $\mathrm{C}$ inf. dextr., $\mathrm{C}$ sup. sin.). 61 (obs. nombreux terriers de renard). 65 ( $\mathrm{M}_{1}$ dextr.). 68 ( $\mathrm{P}^{3}$ sin., crânes).

\section{Fam. URSIDAE}

Ursus arctos L. 1 (fragm. crâne). $\mathbf{2 3}$ (mdb., crâne, cent. pelv. : juv.).

$(\dagger)$ Ursus spelaeus Rosenm. 1 (C). 6 (C). 8 ( $\mathrm{P}_{4}$ sin., $\mathrm{I}_{3}$ dextr., $\left.\mathrm{I}^{2} \sin .\right)$. 16 ( $\mathrm{C}$ et nombreux autres fragments). 17 ( $\mathrm{I}^{3}$ sin. et nombreux os longs dans la croûte stalagmitique). $\mathbf{3 8}$ (fragm. crâne). $\mathbf{4 6}$ (C, nombreux os longs, Vertébres). 48 (metatarsien V dextr., os long). 50 (Canins, os longs). 51 ( $\mathrm{M}^{\mathbf{1}}$ sin., $\mathrm{M}_{1}$ dextr., $\mathrm{C}$ sup. sin.). $\mathbf{5 2}$ (os longs, leg. Tr. Novac). $\mathbf{6 8}$ (divers, mal conservés). $\mathbf{7 1}$ (depôt d'os longs). 72 ( $\mathbf{M}^{1}$ sin. fragm., etc.) Nous avons vu des pièces de Ursus spelaeus, encore inédites, de grottes du Banat, dans le Musée Régional du Banat (5; 18: provenance douteuse), dans le Musée minier d'Anina (12; V. Sencu a aussi trouvé Ursus spelaeus dans cette grotte) et, suivant des renseignements verbaux, on aurait trouvé cette espèce aussi dans 44 et $\mathbf{7 0}$.

Note: dans 69: canin de Ursus sp.

\section{Fam. MUSTELIDAE}

Mustela putorius L. 56. 68.

Vormela peregusna (Guldenstädt). 20 (crâne).

Martes foina (Erxleben). 7 (restes mal conservés). $\mathbf{2 0}$ (mdb. dextr.). 68 (crânes, arc mdb., 2 mdb. dextr.).

3) La date de la récolte n'est pas mentionnée quand il s'agit uniquement de pièces squelettiques; elle est indiquée, par contre, quand il est question d'animaux capturés vivants ou bien d'observations portant sur de tels animaux. Malheureusement, dans quelques cas nous n'avons plus pu indiquer exactement de quelles pièces il s'agissait pour telle ou telle espèce dans telle ou telle station. 
Martes martes L. 68 (mdb. sin. fragm.).

Meles meles (L.). 39 (arc. mdb.). 42 (crâne entier, crâne fragm.). 52 (arc mdb.).

Lutra lutra (L.). 66 (C inf. dextr., C sup. sin.). La grotte est parcourue par un ruisseau qui en sort par une assez grande ouverture.

Note: dans 69 : fragm. mdb. de Mustellidae (indet.).

\section{Fam. HYAENIDAE}

(†) Crocuta spelaea (Goldf.). 33 (max. inf. Au fond d'un petit aven). 34 (fragm. crâne, à même la surface du dépôt de remplissage massif). 68 (fragm. crâne).

\section{Fam. FELIDAE}

Felis catus L. 29 (fragm. carnassier, 2 fragm. canins:, ,cf. catus" ${ }^{\prime)} .34$ (crâne neural).

Felis sylvestris Schreber. 17 (cubitus et radius dextr., fragm. calcaneum sin., tibia sin. et dextr. fragm., bassin fragm.). $\mathbf{2 0}$ (arc mdb.). 56. $\mathbf{5 8}$.

$(\dagger)$ Felis (Lynx) lynx L. 48 (le matériel, recueilli dans l'argile d'un brunroux en surface de dépôts pléistocènes, a été publié par Terzea 1963).

\section{Ord. ARTIODACT YLA}

\section{Fam. SUIDAE}

Sus scrofa L. 7 ( $\mathrm{P}^{4--} \mathrm{M}^{2}$ dextr.). 8 (D $\mathrm{D}^{3-4} \sin ., \mathrm{D}_{2-4} \sin ., \mathrm{M}_{1} \sin$.). 10 (mdb. dextr. fragm.). 30 (I sup.). $\mathbf{5 0}$ ( $\mathrm{M}^{1} \sin$.). Dans les trois premières grottes il s'agit, probablement, du porc domestique; dans la 4-e il s'agit, probablement, du porc sauvage, mais le matériel insuffisant ne permet pas d'affirmer si c'est $S$. scrofa attila ou bien une forme fossile.

\section{Fam. CERVIDAE}

Cerous elaphus L. 5 ( $\mathrm{I}_{1-2}$ sin., $\mathrm{I}_{1-3}$ dextr.).

Capreolus capreolus (L.). 11 (palais avec les séries dentaires). 17 (mdb. sin. fragm., humer. + radius + metacarp dextr.). 51 (humer. dextr. fragm. dist., radius dextr. juv., metatars juv. fragm.). $\mathbf{5 2}$ (M. dextr. fragm.). 68.

\section{Fam. BOVIDAE}

Capra hircus L. $\mathbf{5 1}$ (mdb. sin. fragm., metacarp. sin., falanges).

Capra ibex L. 48 (fragm. coxal).

Rupicapra rupicapra (L.) 5 ( $\left.\mathrm{D}^{2-4} \sin .\right) .7$ ( $\left.\mathrm{D}^{2-4}, \mathrm{M}^{1-2}\right) .20$ ( $\mathrm{M}^{1}$ dextr., $\mathrm{M}^{2-3}$ sin., $\mathrm{M}_{2}$ dextr.).

Note: des pièces squelettiques indéterminables, soit de Ovis soit de Capra, ont été recueillies dans : 1, 20, 21, 22, 41, 63, 67, 68, 69.

Ord. LAGOMORPHA (DUPLICIDENTATA)

\section{Fam. LEPORIDAE}

Lepus europaea Pallas. 19 (max. sup. fragm.). 
Ord. RODENTIA (SIMPLICIDENTATA)

\author{
Fam. SGIURIDAE
}

Sciurus vulgaris L. $\mathbf{3 5}$ (un écureuil avec sa progéniture observé dans la grotte). 50 (mdb. dextr. fragm.). $\mathbf{5 2}$ (fragm. mdb.).

\title{
Fam. MUSCARDINIDAE
}

Glis glis L. 2 (mdb. sin. fragm.). 5. 8.13 (2 mdb., 2 cent. pelv., humerus, femur). 17 (2 fragm. crâne, 7 fragm. mdb.). $\mathbf{2 0}$ (mdb. sin.). 26 (mdb. dextr. sans dentition). 29 (fragm. mdb. dextr.). 30. 41 (fragm. crâne). 46 (4 mdb., 6 I sup., 4 I inf.). 50 (9 mdb., fragm. crâne). $\mathbf{5 1}$ (4 fragm. mdb.). $\mathbf{5 2}$ (6 mdb. sin., 5 mdb. dextr., fragm. crâne). 56 (mdb., crâne). 57. 65 (mdb. sin.). 68 (2 mdb. sin.).

Muscardinus avellanarius L. 6 (fragm. crâne, 3 fragm. max. sup., 2 mdb. sin., mdb. dextr.). 20 (crâne, mandibules). 52 (2 mdb. sin., mdb. dextr.). 57. Dans 20, observé des terriers.

\section{Fam. CRICETIDAE}

(†) Cricetulus migratorius Pallas. 17 (mandibules, dents). $\mathbf{2 7}$ (fragm. mdb.). 65 (mdb. dextr.). On sait que C. migratorius disparut d'Europe Centrale dès la fin du Pleistocène. Hamar (1963b) en a récemment mentionné des pièces trouvées dans des ingluvies d'Oiseaux dans deux stations de Moldavie septentrionale ce qui prouverait la réapparition de cette espèce sur le territoire de Roumanie.

\section{Fam. MICROTIDAE}

Clethrionomys glareolus (Schreber). 5. 16 (mdb. dextr. fragm.). 17 (mdb. sin. fragm.). 20 (2 mdb. sin., mdb. dextr., le tout fragm.). 38. 46 (2 mdb.). 52 (3 mdb. sin., 1 mdb. dextr.). 57. 68 (mdb. dextr.). 72 (mdb. dextr.).

Arvicola terrestris L. 5.

Microtus aroalis (Pallas). 7. 16 (mdb. dextr. fragm.) 20 (2 mdb. sin. fragm.). 40 (mdb. dextr., mdb. sin., les deux fragm.). 52 (3 mdb. dextr., 3 mdb. sin., mdb. sin. fragm.). 57. 58 (fragm. crâne, 2 mdb.). 60 (mdb. dextr.). 65 (fragm. crâne).

Note: des fragments de Microtus (ou d'un autre Microtide), dont l'identification n'a pas été possible, ont été recueillis dans : 20, 27, $4 \mathbf{1}$.

Pithymys subterraneus De Sélys Longchamp. 52 (mdb. sin., fragm. crâne).

\section{Fam. MURIDAE}

Apodemus sylvaticus (L.). 1 (2 fragm. max. sup.). 7. 11 (mdb.). $16\left(\mathrm{M}_{1}\right)$. 17. 20 (6 mdb. sin., 4 mdb. dextr., 11 mdb. fragm.). $\mathbf{3 4}$ (mdb. sin. fragm.). 40 (mdb. sin. fragm.). 41 (mandibules). 46 (2 mdb.). 48 (3 fragm., mdb. sans dentition). 52 (fragm. crâne, 28 mdb. sin., 25 mdb. dextr.). 57. 60 (fragm. crâne). 68 (fragm. reg. maxillaire, crâne, mdb. dextr., mdb. sin.). 72 (4 mdb. sin., 2 mdb. dextr.).

\section{Fam. SPALACIDAE}

Spalax microphtalmus Güld. 46 (mdb. sin.). 
Ord. INSECTIVORA

Fam. SORICIDAE

Sorex araneus L. 38.

Sorex minutus L. ?. 5. Seules des recherches stratigraphiques dans cette grotte pourraient permettre d'affirmer s'il s'agit de matériel fossile ou actuel.

Note: des pièces appartenant probablement à Sorex proviennent aussi de $\mathbf{5 7}$.

Crocidura leucodon Herman. 7. 52 (crâne, 2 mdb.). 66 (dent). Du matériel peu concludent a été recueilli aussi dans $\mathbf{5 7}$.

\section{Fam. TALPIDAE}

Talpa europaea L. 5. 41 (mdb.). 52 (6 mdb. dextr. + sin.). 57.

\section{Ord. CHIROPTERA}

\section{Fam. RHINOLOPHIDAE}

Rhinolophus ferrum-equinum Schreber. 3 (2 crânes, mdb.). 5 (12. XI. 61, coll. expl. vivants, obs. expl. isolés). 8 (pièces ostéologiques; 13. XI. 61, coll. expl. vivants, obs. expl. isolés dans la galerie principale, parasités par Nycteribia biarticulata Herman). 9 (30. IX. 61, coll. expl. vivants, obs. expl. isolés, non parasités). 12 (fragm. crâne; 28. IX. 61, coll. expl. vivants, obs. expl. isolé, inactifs, non parasités, dans la galerie principale et dans les salles et galeries latérales). 13 (5. X. 61, coll. expl. vivants, obs. expl. isolés, inactifs, non parasités). 17 (2 fragm. crâne, nombreux cadavres; 3. X. 61, coll. expl. vivants, obs. expl. isolés, jamais des colonies). 18 (crâne; 1.VII.61, obs. expl. isolés, actifs; 2. X. 61, coll. expl. vivants, obs. partout des expl. isolés, inactifs, non parasités, ainsi qu'une colonie de 500 expl. environ au point de station $48 ; 24$. X. 65 , obs. colonie de 20 expl. au point de station 52 et expl. isolés dans la galerie subfossile). $\mathbf{2 0}$ (26. IX. 65, obs. dans les deux galeries expl. isolés, inactifs ou peu actifs, non parasités). 24 (crâne, 2 mdb.). 30 (19. XI. 61, obs. expl. isolés, non parasités; 6. X. 63, obs. expl. isolés, actifs). $\mathbf{3 1}$ (30. VII. 64, obs. expl. actifs). 36 (6. X. 65, obs. expl. non parasité). 40 (fragm. crâne; 21. X. 55, obs. colonie d'individus actifs à $200 \mathrm{~m}$ depuis l'entrée; 1. VIII. 63, obs. colonie d'individus très actifs, au point de station $40 ; 9$. X. 63, obs. colonie de plusieurs centaines d'expl. moins actifs, parasités par Nycteribia biarticulata Hermann et des Ixodidae (Ixodides vespertilionarum?) au point de Station 36$) .43$ (2 crânes, mdb.; 25 . IX. 61 , coll. expl. vivants, obs. individus isolés et colonie formée de plusieurs groupes de 10-20 expl.). 46 (crâne). 47 (fragm. crâne, fragm. mdb.). 48 (2 mdb.). 49 (crâne, 4 mdb.; 10. X. 61., 1. X. 63, oks. expl. isolés, non parasités). 51 (fragm. crâne, 9 mdb.; 9. X. 61, obs. expl. actifs). 52 (2 fragm. crânes, 4 mdb.; dans la "Salle des Chauves-souris», grande colonie de plusieurs centaines d'exemplaires actifs, obs. le 12. X. 61 ; à 22. IX. 62, la grande colonie n'existait plus, il y avait seulement de petits groupes; à 5. X. 63, colonie de nouveau présente, composée d'expl. actifs). 56 (matériel ostéologique). $\mathbf{5 7}$ (riche matériel ostéologique). 58 (3 fragm. crânes; 9 . VI. 62, coll. expl. vivants, obs. petites colonies et expl. isolés). 62 (crâne, mdb. dextr.; 10. VI. 62, coll. expl. vivants, obs. expl. isoles, parasités par Penicil- 
lidia dufouri Westw., Nycteribia schmidli Schiner, N. biarticulata Herm., Acariens parmi lesquels l'Ixodide Ixodes chiropterorum Bab. \& Jan.; mais les Nycteribiides ont été pris aussi sur Miniopterus schreibersi et sur Myotis myotis). 64 (20. VI. 62, obs. expl. isolés, actifs). 72 (mdb. sin.).

Rhinolophus hipposideros Bechstein. 4 (25. VI. 63. coll. expl. vivants, obs. expl. isolés, actifs, non parasités). 5 (12. XI. 61, coll. expl. vivants, isolés). 7 (15. XI. 61, obs. quelques expl.). 8 (13. XI. 61, coll. expl. vivants, isolés, dans la galerie principale; 20. V. 65, obs. expl. isolé, actif). 13 (5. X. 61, coll. expl. vivants, isolés, inactifs). 15 (6. X. 61, obs. un exemplaire). 17 (crâne, etc.). 18 (2. X. 61, coll. expl. vivants, obs. expl. isolés, non parasités). 23 (4. X. 65, obs. un expl. non parasité). 25 (17. V. 65, obs. un exemplaire actif). 46 (crâne). 48 (11. X. 61, observation). 49 (10. X. 61, obs. expl. isolés). 50 (11. X. 61, observation). 52 (12. X. 61, obs. expl. isolés). 54 (7. VI. 62, obs. expl. parasité par Nycteribia biarticulata Herm.). 57 (riche matériel ostéologique). 59 (9. VI. 62, obs. expl. non parasité). 67 (crâne, 2 mdb.).

Rhinolophus mehelyi Matschie. 10 (mat. ostéol.). 38 (mat. ostéol.). 46 (mdb. sin, mdb. dextr.).

Rhinolophus euryale Blasius. 40 (crâne, max. inf.).

Rhinolophus blasii Peters. 5 (mat. ostéol.). 38 (17. XI. 61, coll. expl.vivants, obs. expl. isolés, non parasités). 40 (mdb.). 52 (crâne, 3 mdb.). 53 (crâne). 56 (mat. ostéol.). 58 (9. VI. 62, coll. expl. vivants, obs. petites colonies et expl. isolés, parasités: Nycteribia biarticulata Herm., Ixodes vespertilionis Koch, Aphaniptera indet.). 62 (3 crânes, 6 mdb.; 10. VI. 62, coll. expl. vivants, obs. expl. isolés). 68 (mat. ostéol.). 70 (crâne). 72 (6 crânes, 4 mdb. dextr.).

\section{Fam. VESPERTILIONIDAE}

Myotis myotis Borkhausen. 1 (11 crânes, 16 mdb., 2 mdb. juv.). 12 (2 crânes, 6 mdb.). 13 (5. X. 61, coll. expl. vivant, parasité par des Acariens). 16 (29. IX. 65, coll. expl. vivant, obs. expl. isolés assez alertes; parasites: Spinturnicidae indet.). 17 (5 crânes, etc.). 18 (fragm. mdb., etc.; 24. VII. 63, coll. expl. vivant, parasité par Nycteribia latreillii Leach, Penicillidia dufouri Westw.). 24 (4 crânes, 7 mdb.). 29 (mdb. et dents ; 25. VII. 63, coll. expl. vivants dans des colonies mixtes; obs. nombreuses colonies, soit formées seulement de M. myotis (juv., point de Station 21), soit mixtes; des centaines d'expl. dans chaque colonie; les Chauves-souris sont fort parasitées: Nycteribia latreillii Leach, N. schmidli Schiner, Penicillidia dufouri Westw., obs. aussi Acariens et Aphaniptères). 30 (mat. ostéol.). 40 (mdb.) 46 (humerus). 52 (7 mdb.). 62 (2 crânes avec dentition lactéale, 1 fragm. crâne, 6 mdb.; 10. VI. 62, coll. expl. vivant; obs. deux colonies de quelques centaines d'expl. chacune, dans la galerie à eau; pour les parasites, voir la mention faite pour cette grotte sous Rhinolophus ferrum-equinum; à 23. VI. 65, la colonie formée de quelques centaines d'expl. actifs, était présente au mème endroit). 68 (crâne, mdb.). 70 (crâne, mdb. sin.). 72 (fragm. mdb. sin.).

Myotis oxygnathus Monticelli. 12 (28. IX. 61, coll. expl. vivants, obs. expl. isolés, inactifs, partout dans la galerie principale et dans les latérales; parasites : Nycteribia latreillii Leach, Penicillidia dufouri Westw., Acariens indet.). 13 (crâne, 2 mdb.). 18 (2. X. 61, coll. expl. vivants, obs. expl. isolés, parasités par des Acariens indet.). 24 (2 crânes). 44 (crâne). 46 (19. VIII. 62, 
coll. expl. vivant, obs. peu nombreux expl. isolés, fort actifs). 51 (3 mdb.). 52 (crâne, 2 mdb.). 71 (fragm. mdb. sin.).

Myotis bechsteinii Kühl. $\mathbf{1 7}$ (crâne; peut-être d'autres pièces squelettiques aussi).

Myotis capaccinii Bonaparte. $\mathbf{2 0}$ (3 mdb.). 23 (mdb.). $\mathbf{2 4}$ (1 mdb., crâne). 29 (25. VII. 63, coll. expl. vivants, obs. dans des colonies mixtes avec M. myotis et Miniopt. schreibersi; parasités par Nycteribia pedicularia Latr. et Penicillidia dufouri West.

Myotis emarginatus Geoffroy. 17 (5 crânes, etc.). 24 (crâne). 62 (crâne). 63 (mdb. sin.).

Myotis mystacinus Kühl. 2 (mdb.). 16 (29. IX. 65, coll. expl. vivant, obs. expl. isolés, vivants, parasités par des Spinturnicidae). 17 (crâne, etc.).

Nyctalus noctula Schreber. 68 (2 crânes, 1 fragm. crâne, 7 mdb.).

Miniopterus schreibersi Kühl. 1 (crâne; 22. V. 63, coll. expl. vivants, obs. expl. isolés, inactifs, parasités par Nycteribia schmidli Schiner, Penicillidia dufouri Westw. et Acariens). 3 (23. V. 63, coll. expl. vivant, obs. seulement expl. isolés, parasités par des Acariens indet.). 9 (crâne). 29 (4 crânes, mdb.; 25. VII. 63, coll. expl. vivant dans une colonie mixte avec $M$. myotis et Miniopt. capaccinii; obs. d'autres grandes colonies mixtes; aux points de Station 34 et 36 à 25. VII. 63 et 31. VIII. 64, obs. colonies de jeunes de Miniopt. schreibersi; parasites: Nycteribia schmidli Schiner, Penicillidia conspicua Speiser, P. dufouri Westw.). 40 (mdb.; une colonie de Minioptères (?) actifs a été observée le 9 . X. 63 près de celle de Rhinolophes). 45 (2 mdb., 2 crânes, 1 crâne juv.). 56 (mat. ostéol.). 62 (3 crânes, 2 mdb. dextr.; 10. VI. 62 , coll. expl. vivant, obs. groupes de plusieurs expl.; pour les parasites, voir la mention de cette grotte sous Rhinolophus ferrum-equinum). 63 (2 crânes, mdb. dextr.).

Plecotus auritus L. 52 (5 mdb.). 68 (mdb.).

Plecotus austriacus Fischer ?. 29 (mdb.).

Barbastella barbastellus Schreber. 17 (2 crânes, etc.). 68 (crâne, mdb.).

Eptesicus serotinus Schreber. 63 (mdb. sin.). 68 (mdb. sin., mdb. dextr.). Eptesicus nilssoni Keyserl. et Blasius. 17 (crâne).

Pipistrellus pipistrellus Schreber. 24 (2 crânes). 30 (mat. ostéol.). 68 (20 crânes). 69 (crâne).

Note: dans plusieurs grottes, des colonies plus ou moins importantes de Chauves-souris ont pu être observées sans que des exemplaires puissent en être récoltés (ou bien le matériel collecté avait été perdu avant d'être identifié).1: colonie de quelques centaines d'expl. probablement Rhinolophes, dans la salle finale, obs. 12. V.63. 12: colonie obs. le 15. VII. 61, dans «l'avencheminée" près l'entrée par la doline. 20: colonie de quelques dizaines d'expl. actifs d'un Myotis, obs. le 26. IX. 65. 45: à 20. VI. 60, obs. colonie de 60 expl. sur la voûte à $3 \mathrm{~m}$ de hauteur; et le 17. VIII., obs. nombreux expl., mais isolés. 57: colonie de 400-500 expl. dans la coupole de la grande salle, à $25 \mathrm{~m}$ de hauteur ; formée de rhinolophes, la colonie est pratiquement inaccessible; obs. aussi, à $10 \mathrm{~m}$ depuis l'extrée, une colonie de quelques dizaines d'expl. 68: obs. plusieurs colonies, formées de dizaines ou de centaines d'expl., en juin, juillet, août, en divers endroits de la grande salle du système III.

Le matériel ostéologique et les animaux vivants que nous avons pu recueillir dans 70 grottes environ, a permis la détermination, avec une précision absolue, de 53 espèces de Mammifères fossiles et actuels. Il 
s'agit de 14 Carnivores, 6 Artiodactyles, 1 Lagomorphe, 10 Rongeurs, 3 Insectivores et 19 Chiroptères. Mentionnons, à titre d'éléments présentant un intérêt particulier d'un point de vue ou d'un autre: $\dagger$ Crocuta spelaea, $\dagger$ Felis lynx, Capra ibex, $\dagger$ Cricetulus migratorius, Rhinolophus mehelyi, Myotis bechsteini, Eptesicus nilssoni.

\section{RÉSUMÉ}

Dans 70 grottes environ du Banat, les auteurs ont rassemblé une collection de documents (matériel ostéologique et animaux vivants) se rapportant aux Mammifères, fossiles et actuels. Une liste des grottes est présentée et on donne pour chaque grotte l'énumération des formes signalées. Dans la seconde partie, on donne pour chaque espèce: les grottes ayant fourni le matériel, l'inventaire du matériel ostéologique, les observations faites sur les animaux vivants (et surtout sur les Chiroptères). 53 espèces de Mammifères fossiles et actuels ont pu être déterminées avec une précision absolue (dont 14 Carnivores, 6 Artiodactyles, 1 Lagomorphe, 10 Rongeurs, 3 Insectivores, 19 Chiroptères).

\section{SUMMARY}

The authors assembled from about 70 caves a rich collection of osteological material and specimens of living or fossil mammals. A list of the caves is given with an enumeration of the identified species for each cave. Under each species the caves which supplied the material are listed. This is followed by an inventory of the osteological material and by observations on the living animals (especially bats). Fifty-three mammal species (fossil and living) were accurately determined (14 carnivores, 6 artiodactyls, 1 lagomorph, 10 rodents, 3 insectivores, and 19 bats).

\section{BIBLIOGRAPHIE}

Balogh, E. (1937) - A Cuptorul Porcului barlang. MS.

- (1938a) - Fiutal barlangi medve (Ursus spelaeus Ros.) maradvániok a Popováci barlangból. Erdélyi Muzeum, t. XLIII.

- (1938b) - A nyest (Mustela foina) mint barlangi ragadozó. Erdélyi Muzeum, t. XLIII.

- (1940) - Rendellenességek barlangi medve (Ursus spelaeus Ros.) fogakon és csontokon. Erdélyi Muzeum, t. XLV, nr. 2.

- (1942a) - Ujadat a nyest élatmódjának ismeretéhez. Allatani Közlemények, t. XXXIX.

- (1942 b) - Osemberi maradvániok a Bánságihegyvidék két barlangjából. Közlemények az Erdélyi Nemzeti Muzeum Ėrem - és Régiségtárából, t. II.

- (1956) - A Popováci barlang. MS.

- (1957) - A Comárniki barlang teljes bépe. MS.

BокоR, E. (1921) - A magyarhoni barlangok izettlábui (Arthropoden der ungarischen Grotten). Barlangkutatás, t. IX, nr. 1-4.

Botoşaneanu, L., Negrea, A., et Negrea, St. (1966) - Grottes du Banat explorées de 1960 à 1962. Paris, C.N.R.S.

Galinescu, R. (1931) - Verzeichnis und Bibliographie der Säugetiere Rumäniens. Ztschr. für Säugetierkunde, t. 6, nr. 2. 
Dumitrescu, M., Tanasachi, J., et Orghidan, T. (1962-63) - Rǎspîndirea Chiropterelor în R. P. Română. Lucr. Inst. de speol. ,,Emil Racoviță“, t. I-II.

Halaváts, J., (1888) - Sztirnik barlang (Resica vidékén) emlös maradnányai. Természettudományi Köslöny, t. XX.

Hamar, M., (1963a) - Contributions to the study of the upper pleistoceneholocene fauna of small mammals (Chiroptera, Insectivora, Glires). Revue de Biologie, Académie de la R.P.R., t. VIII, nr. 2.

- (1963b) - Die Anwesenheit von Cricetulus migratorius (Pall. 1773) in Gewöllen von Asio otus (L.) und Athene noctua (Scop.) in Rumänien. Acta Theriol., t. VI, nr. 11.

Hoernes, R. (1875) - Vorlage von Wirbeltierresten (Ursus spelaeus und Capra ibex) aus der Bohuj-Höhle bei Anina. Verh. der k. k. Geol. Reichsanstalt.

KADIč, O. (1917) - Barlangi Közlemények, t.V.

Maxim, I. (1943) - La Chèvre des rochers "Ibex" dans le pléistocène de Roumanie. C. R. des Sèances de l'Acad. Sci. Roumanie, t. VII.

Méhely, L. (1900) - Monographia Chiropterorum Hungariae. Budapest.

Negrea, St., Negrea, A., Sencu, V., et Botoşaneanu, L. (1965) - Grottes du Banat (Roumanie) explorées en 1963. Int. Journ. Speleology, t. I, part. 4 .

Nicolaescu-Plopşor, C.S., et Mateescu, C. N. (1955) - Santierul arheologic Cerna-Olt. Stud. Cerc. Istorie Veche, t. VI, nr. 3-4.

Orghidan, T., Puşcariu, Val., Bleahu, M., Degu, V., Rusu, T., et Bunescu, A. (1965) - Harta regiunilor carstice din România. Lucr. Inst. de Speol. ,,Emil Racovițǎcc, t. IV.

Orтhмаул, T. (1872) - A barlangok paläontologiai és történeti jelentösége, tekintettel Magyarország, de különösen Délmagyarország barlangjaiva. A magyar orvosok és termeszetvizsgálok 1872 ben Herkules fürdön tartott vándorgyülésének munkálatai.

Paszlawski, J. (1918) - Mammalia. In: Fauna Regni Hungariae I. Budapest Petényi, S. J. (1880) - Carnivora, Chiroptera. Termés. Füsetek, t. 4.

Primics, G. (1890) - Spuren des Höhlenbären (U. spelaeus Blumenb.) in Ungarn. Földtani Köslony, t. XX, nr. 5-7.

Sencu, V. (1963) - Cercetări asupra carstului din jurul localitătii Anina (Banat); peşterile din bazinele pîraielor Anina şi Bohui. Probleme de Geografie, t. X.

Teglas, G. (1883) - A Buhuj (Bagolyvár) nevii scontbarlangvól. Természettudományi Közlöny, t. XV.

- (1884) - A Buhuj (Bagolyvár) nevii csontbarlang Stajerlak-Anina hatávában. A Magyar Tudományos Akadémia Math. es Term. Közleményi, t. XIX.

Terzea, El. (1963) - Observații asupra morfologici lui Felis (Lynx) lynx L. foss. şi cîteva considerații asupra răspîndirii speciei pe teritoriul R. P. R. Stud. Cerc. biol. Acad. R. P. R., ser. biol. anim., t. XV, nr. 3. 